\title{
Electroencephalographic changes as prognostic indicators after psychosurgery
}

\author{
B IDI M EVANS,* P K BRIDGES, $\dagger$ AND J R BARTLETT † \\ From the Department of Cinical Neurophysiology* and the Geoffrey Knight Psychosurgical Unit, $\dagger$ \\ Brook General Hospital, London
}

SUMMARY Thirty-five patients were studied by EEG with recordings taken the day before, two weeks after, and six months after psychosurgery. Most showed a characteristic frontal slow activity of varying degrees after operation with varying enhancement of background activity. The amount and spread of the frontal slow waves two weeks after operation showed a significant positive correlation with the clinical outcome one year later, which suggests a relatively objective and very early indication of the subsequent clinical response to psychosurgery.

This was a prospective study carried out in order to investigate whether there are changes in the EEG that might be associated with stereotactic subcaudate tractotomy. This psychosurgical operation, devised by Knight, ${ }^{1}$ with clinical results reported by Ström-Olsen and Carlisle ${ }^{2}$ and by Göktepe $e t a^{3}$ involves the stereotactic placement of bilateral lesions produced by an array of radioyttrium rods $\left({ }^{90} \mathrm{Y}\right)$ in the ventromedial quadrants of the frontal lobes. The site and size of the lesions have been discussed by Newcombe ${ }^{4}$ and Bartlett and Bridges. ${ }^{5}$

\section{Patients}

Patients consecutively admitted after acceptance for psychosurgery were included provided they were under 75 years old, they had not previously had psychosurgery or a course of electro-convulsive therapy within the previous six months, and there were no neurological abnormalities present likely to produce EEG changes. With these criteria 35 patients were studied with an age range from 26-72 years, of whom 24 were female and 11 were male. The large majority of the patients had intractable depressive illnesses, while some presented with severe obsessional neuroses or with incapacitating anxiety or tension.

Address for reprint requests: Dr PK Bridges, Brook General Hospital Shooters Hill Rd, London SE18 4LW.

Accepted 10 March 1981

\section{Methods}

The records were performed with SLE E18/16 machines using silver/silver chloride electrode discs applied with collodion according to the method suggested by Pampiglione, ${ }^{6}$ but with paired occipital electrodes. All records included 3 minutes of hyperventilation and intermittent photic stimulation with an SLE stroboscope.

Three records were performed on each patient: 1st record: This was taken on the day before operation. In all cases medication was withdrawn at least one week before the operation and replaced by diazepam $5 \mathrm{mg}$ thrice daily with nitrazepam at night if needed. In the case of five patients, one additional drug was continued pre-operatively, namely for each separate patient-nortriptyline, medazepam, clorazepate, perphenazine, chlorpromazine.

2nd record: This was carried out two weeks after operation, just before discharge from the Unit in most cases. All patients continued after the operation on diazepam and nitrazepam alone, except for five who at this time were each having one of the following in addition-medazepam, perphenazine, clorazepate and two patients were receiving chlorpromazine. $3 r d$ record: This was taken six months after operation when the patients returned to the Unit for a routine follow-up interview in order to assess their progress. For this investigation they had been asked to stop other forms of medication one week before their attendance and they then took only diazepam and nitrazepam.

The EEG records were all assessed by one author 
(BME) before the patients attended one year after operation for a decision as to the clinical outcome, and this author was not involved with the clinical interview.

The formal clinical assessment of response to the operation was carried out at one year by a senior psychiatrist otherwise not associated with the Unit. This is done at one year because there is a wellrecognised delayed response to the operation, the effects of which are not usually immediate, and there is more often a slow improvement over 3-6 months or even longer. At one year the patients were classified by the independent psychiatrist as follows: ${ }^{3}$

I Recovered, no symptoms and no treatment required.

II Well; mild residual symptoms, little or no interference with daily life.

III Improved but significant symptoms remain which interfere with the patient's life.

IV Unchanged.

V Worse.

We report the results in terms of categories I and II together because there is evidence to show, on several parameters, that there is no clinical difference between these groups. ${ }^{3}$

\section{Results}

\section{EEG FINDINGS}

1st record: The first records were normal apart from an excess of fast activity in 18 cases, attributable to medication with benzodiazepines. Some degree of abnormality was present with 17 patients. Of these, 12 showed only an increase of rhythmic theta as well as fast activity, and this too was probably related to medication. Five records showed some episodic changes. These were brief episodes of higher voltage activity in the theta range and associated with sharp components seen over the mid-temporal and anterior temporal regions of both hemispheres. Two patients also had an increase of slow activity on overbreathing in addition to this.

2nd record: In only three patients was there no change in the second record. For 32 patients there was slow activity seen over the frontal regions (fig). This activity was irregular, at frequencies between 1 and $3 \mathrm{~Hz}$ and unaffected by eye opening. Eye movements were monitored in all patients after the slow activity was recognised, with
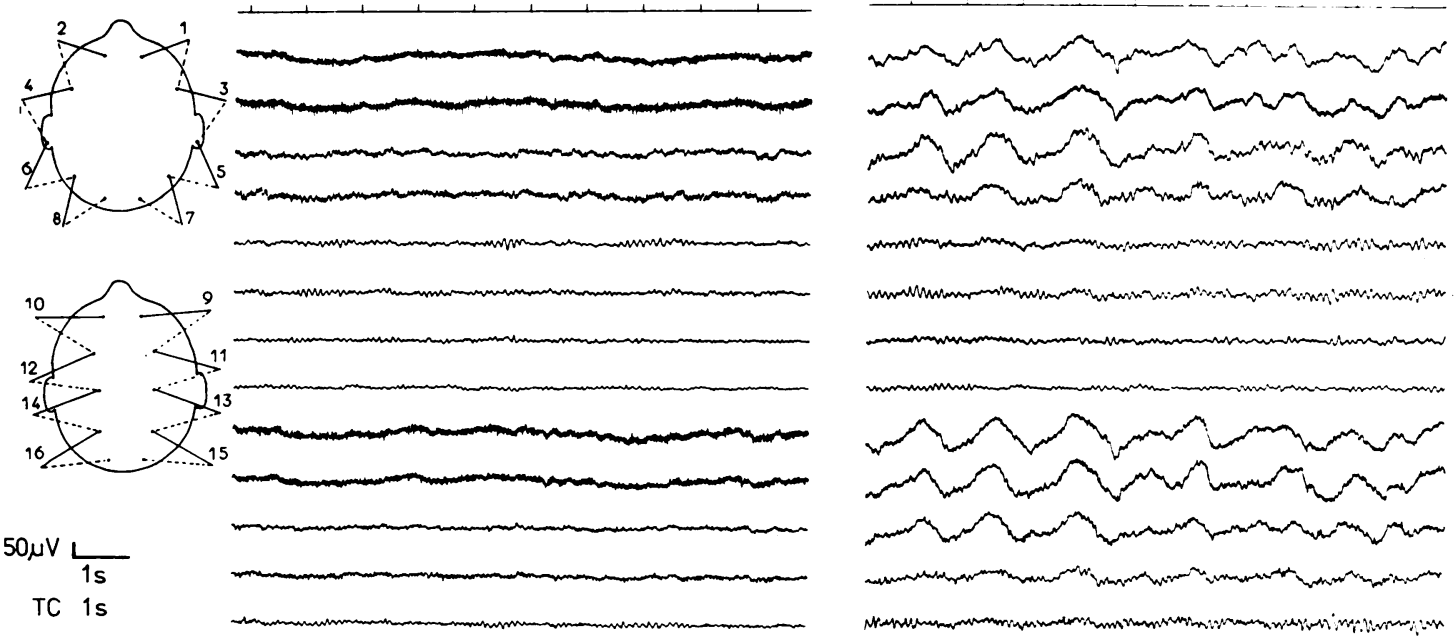

Figure EEG records from a 36-year-old woman (a) before and (b) 18 days after subcaudate tractotomy. In the record after operation (Group 4) marked bi-frontal slow activity is present with some enhancement of background activity. 
electrodes at the outer canthus and below each eye. This made it clear that the potentials were cerebral in origin. In eight patients, in addition to the frontal slow, there was a diffuse excess of slow activity spreading backwards as far as the occipital regions. In a further three the slow activity was less well localised, the changes consisting of diffuse slow waves in all areas at frequencies between 2 and $3 \mathrm{~Hz}$ with only slight frontal preponderance.

In addition to the slow waves, 17 patients showed a change in the background activity. This was an accentuation of the alpha rhythm which became more abundant, more widespread and of higher voltage. In those patients where the background activity was in the theta range it was this frequency which was accentuated.

In view of the wide variation in the records at 2 weeks they were divided into six groups:

(1)-no slow waves (3 patients), (2)-minimal slow waves (5), (3)-moderate slow waves of low voltage localised to the frontal regions (11), (4)marked high voltage slow waves, localised to the frontal regions (5), (5)-marked high voltage slow waves, localised to the frontal regions with spread of slow to other areas (8), (6)-generalised slow with poor localisation (3).

3rd record: Follow-up records at six months were obtained for twenty-seven patients of whom thirteen had been taking antidepressant medication which they had not been receiving during their admission for operation. In five cases there was still some trace of frontal slow present; all but one of these patients had originally been in groups 4,5 or 6 , and there was a marked decrease in the amount of slow waves seen. One case was in group 2 and here the record was the same at six months as it had been two weeks after operation. The accentuation of background activity was still present at six months in four patients and only one case showed both slow activity and background change at six months.

\section{CLINICAL AND EEG RELATIONSHIPS}

Of the thirty-five patients in the study, ten $(29 \%)$ were assessed one year after operation as having responded well (categories I and II), eleven (31\%) were improved (III) and fourteen (40\%) failed to respond (IV). No patient was found to be worse (V). The clinical outcome assessments at one year were compared with the appearances of the second EEG records at two weeks (table). Eight of the ten patients in clinical categories I and II were found in EEG groups 4,5 or 6 and only two in EEG groups 1,2 or 3 . Of the patients who improved (III) there were approximately half in each
Table Clinical outcome related to the degree of EEG disturbance

\begin{tabular}{lccc}
\hline Clinical outcome & \multicolumn{3}{c}{ EEG groups at 2 weeks after operation } \\
\cline { 2 - 4 } I yr after operation & $1-3$ & $4-6$ & Totals \\
\hline I and II & 2 & 8 & 10 \\
III & 5 & 6 & 11 \\
IV & 12 & 2 & 14 \\
Totals & 19 & 16 & 35 \\
\hline
\end{tabular}

For explanation of classifications see text.

of the EEG groups 1-3 and 4-6. A chi-squared test on the data in the table showed statistically significant differences (chi-squared $=10.65$; df 2 ; $p<0.01)$. These data meet the criteria suggested by Siegel $^{7}$ for reliable results with chi-squared testing.

\section{Discussion}

No EEG studies have previously been reported following stereotactic tractotomy although there have been papers in the past on recordings carried out after standard pre-frontal leucotomy, and for the subsequent modified operations which were used up to the 1960 s. $^{8}$ Many workers described frontal slow activity after the older operations, as in the present study, which disappeared in subsequent months. ${ }^{9-17}$ Less frequently have changes in background activity been commented on. ${ }^{11} 1415$ It is notable that the more limited and much more precisely placed lesions now in use have produced almost identical changes in the EEG as did the earlier extensive operations. Furthermore, the degree of change in EEG recordings in relation to subsequent clinical outcome after electroconvulsive therapy has been previously noted ${ }^{18}$ and these EEG findings have been discussed in relation to both ECT and prefrontal leucotomy. ${ }^{19}$ Of course the frontal slow waves might be attributed to cerebral trauma at the time of operation although specificity of response is suggested by the association between the EEG recordings and subsequent clinical outcome especially as this confirms earlier reports.

In conclusion, stereotactic tractotomy seems unlikely to produce a satisfactory psychiatric result at one year unless EEG changes occur at least to the degree of group 4 (marked high voltage bifrontal slow waves) two weeks after operation.

We thank Dr A Norton, Honorary Consultant Psychiatrist, Bexley Hospital, who carried out the clinical assessments at one year. 


\section{References}

1 Knight G. Stereotactic tractotomy in the surgical treatment of mental illness. J Neurol Neurosurg Psychiatry 1965; 28:304-10.

2 Ström-Olsen R, Carlisle S. Bifrontal stereotactic tractotomy. Br J Psychiatry 1971; 118:141-54.

3 Göktepe EO, Young LB, Bridges PK. A further review of the results of stereotactic subcaudate tractotomy. Br J Psychiatry 1975; 126:270-80.

4 Newcombe R. The lesion of stereotactic subcaudate tractotomy. Br J Psychiatry 1975; 126: $478-81$.

5 Bartlett JR, Bridges PK. The extended subcaudate tractotomy lesion. In: Sweet WH, Obrador S, Martin-Rodriguez JG, eds. Neurosurgical Treatment in Psychiatry, Pain and Epilepsy. Baltimore: University Park Press 1977.

6 Pampiglione G. Some anatomical considerations upon electrode placement in routine EEG. Proc Electroenceph Technol Ass 1956; 7(1): 20-30.

7 Siegel S. Nonparametric statistics. 1956; McGraw-Hill, New York.

8 Bridges PK. Bartlett JR. Psychosurgery: Yesterday and Today. Br J Psychiatry 1977; 131:249-60.

9 Davis PA. Electroencephalographic studies on three cases of frontal lobotomy. Psychosom Med 1941; 3:38-50.
10 Hulton EL. Early results of prefrontal leucotomy. Lancet 1941; 2:3-7.

11 Cohn R. Electroencephalographic study of prefrontal lobotomy. Arch Neurol 1945; 53:283-8.

12 Stevens H, Mosovich A. Clinical and EEG investigation of prefrontal lobotomy patients. $\mathrm{Am}$ J Psychiatry 1947; 104:73-80.

13 Mettler FA, ed. Selective partial ablation of the frontal cortex: A correlative study of its effects on human psychotic subjects. New York: Paul B Hoebner 1949.

14 Levin S, Greenblatt M, Healey M, Solomon HG. EEG effects of bilateral prefrontal lobotomy. Am J Psychiatry 1949; 106:174-84.

15 Kreuger EG, Wayne HL. Clinical and EEG effects of prefrontal lobotomy and topectomy in chronic psychoses. Arch Neurol 1952; 67:661-71.

16 Henry CE. Effect on the EEG of transorbital lobotomy. Electroencephalog Clin Neurophysiol 1950; 2:187-92.

17 Hauri P, Hawkins DR. Human sleep after leucotomy. Arch Gen Psychiatry 1972; 26(5) 469-73.

18 Fink M, Kahn RL. Relation of electroencephalographic delta activity to behavioral response to electroshock. Arch Neurol 1957; 78:516-25.

19 Roth M, Kay DWK, Shaw J, Green J. Prognosis and pentothal induced electroencephalographic changes in electroconvulsive treatment. Electroencephalog Clin Neurophysiol 1957; 9:225-37. 